\title{
The Relationship Between Bone Mineral Density Values and Prognostic Nutritional Index As Well As Serum Trace Element Levels in Postmenopausal Women
}

\author{
Postmenapozal Kadınlarda Kemik Mineral Yoğunluğunun Prognostik Nutrisyonel Indeks \\ ve Eser Elementler ile Olan Ilişkisi
}

\author{
(D) Ayhan Kul, (D) Zafer Bayraktutan*, (D) Muhammet Çelik* \\ Atatürk University Faculty of Medicine, Department of Physical Medicine and Rehabilitation, Erzurum, Turkey \\ *Atatürk University Faculty of Medicine, Department of Medical Biochemistry, Erzurum, Turkey
}

\section{Abstract}

Objective: The relationship between bone mineral density (BMD) values and nutritional status as well as serum copper (Cu), zinc (Zn), selenium ( $\mathrm{Se}$ ) and manganese ( $\mathrm{Mn}$ ) levels in postmenopausal women has not been fully elucidated. Therefore, this study aimed to investigate whether there is a relationship between BMD values and nutritional status as well as serum $\mathrm{Cu}$, $\mathrm{Zn}$, Se and Mn levels by evaluating the nutritional status of postmenopausal women based on the BMD values using an objective index.

Materials and Methods: The study included 105 postmenopausal women who were divided into the following 3 groups: Controls (control group, $n=30$, T-score $>-1.0$ ), patients with osteopaenia (osteopaenia group, $n=30, T-s c o r e ~>-2.5$ ) and patients with osteoporosis (osteoporosis group, $n=45$, T-score $\leq-2.5$ ). Moreover, the nutritional status of the patients was determined using the prognostic nutritional index (PNI), which is calculated based on the serum albumin levels and total lymphocyte count. Further, serum Cu, Zn, Se and Mn levels were also determined. The relationship between BMD and PNI as well as the serum levels of $\mathrm{Cu}, \mathrm{Zn}$, Se and Mn was investigated.

Results: The mean age and body mass index (BMI) of the participants were found to be similar in all the groups [control group (age, $63.4 \pm 5.2$ years; BMI, $33.7 \pm 5.6 \mathrm{~kg} / \mathrm{m}^{2}$ ); osteopaenia group (age, $64.6 \pm 5.3$ years; BMI, $33.5 \pm 5.9 \mathrm{~kg} / \mathrm{m}^{2}$ ) and osteoporosis group (age, $65.8 \pm 5$ years; $\left.\mathrm{BMI}, 30.9 \pm 7.2 \mathrm{~kg} / \mathrm{m}^{2}\right)$ ]. In the osteoporosis group, the PNI and serum albumin, Cu and Zn levels were significantly lower than those in the control group $(p<0.05)$, and a weak positive correlation was observed between lumbar total BMD values and PNI as well as serum albumin and $\mathrm{Mn}$ levels $(\mathrm{p}<0.05)$. Furthermore, a positive moderate correlation between PNI and serum Zn levels was observed in the osteoporosis group ( $p=0.045, r=0.063)$.

Conclusion: Low serum albumin levels and a low PNI, particularly in postmenopausal patients with osteoporosis, may have an adverse effect on the total BMD in the lumbar region. Furthermore, Zn supplementation in patients with osteoporosis having low PNI can be a potential additional treatment strategy. $\mathrm{Cu}$ and $\mathrm{Mn}$ supplementation may also provide an additional benefit to existing treatments.

Keywords: Postmenopausal osteoporosis, prognostic nutritional index, trace elements

\section{Öz}

Amaç: Postmenopozal kadınlarda kemik mineral yoğunluğunun (KMY) nutrisyonel durum ve serum bakır (Cu), çinko (Zn), selenyum (Se) ve manganez $(\mathrm{Mn})$ seviyeleri ile olan ilişkisi tam olarak aydınlatılamamıştır. Bu nedenle postmenopozal kadınların KMY değerlerine göre nutrisyonel durumunu objektif bir indeks ile değerlendirerek $\mathrm{Cu}, \mathrm{Zn}$, Se ve Mn ile arasında bir ilişki olup olmadığını incelemeyi amaçladık.

Gereç ve Yöntem: Çalışmada postmenopozal olan 105 kadın üç gruba ayrıldı; kontroller (kontrol grup, n=30, T-skoru >-1,0), osteopenik hastalar (osteopenik grup, $n=30$, T-skoru $>-2,5$ ) ve osteoporotik hastalar (osteoporotik grup, $n=45, T-s c o r e ~ \leq-2,5)$. Hastaların nutrisyonel durumlarının belirlenmesinde serum albümin ve total lenfosit sayısını içeren prognostik nutrisyonel indeks (PNI) kullanıldı. Cu, Zn, Se ve Mn eser elementlerinin serum seviyeleri belirlendi. KMY ile PNI ve eser elementlerin serum seviyeleri arasındaki ilişki incelendi.

Bulgular: Katılımcıların ortalama yaş ve vücut kitle indeksi (VKi) tüm gruplarda benzerdi. [Kontrol grubu (yaş, 63,4 $\pm 5,2 \mathrm{yll} ; \mathrm{VKi}, 33,7 \pm 5,6 \mathrm{~kg}$ /

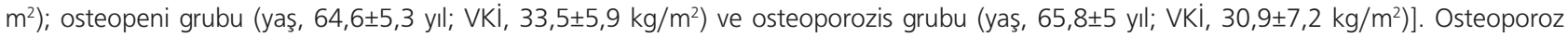
grubunda; PNI, albümin, serum Cu ve Zn seviyelerinin kontrol grubuna göre anlamlı şekilde daha düşük olduğu $(p<0,05)$ ve lomber total KMY değerleri ile PNI, albümin ve serum Mn seviyesi arasında pozitif zayıf bir korelasyon olduğu bulundu $(p<0,05)$. Ayrıca osteoporoz grubunda PNI ile serum Zn seviyesi arasında pozitif orta düzeyde bir korelasyon olduğu tespit edildi ( $p=0,045, r=0,063)$.

Address for Correspondence/Yazışma Adresi: Ayhan Kul Ph.D., Atatürk University Faculty of Medicine, Department of Physical Medicine and Rehabilitation, Erzurum, Turkey Phone: +90 4423446666 E-mail: drayhankul@gmail.com ORCID ID: orcid.org/0000-0003-1313-9469 Received/Geliş Tarihi: 04.08.2020 Accepted/Kabul Tarihi: 20.10.2020

${ }^{\circ}$ Copyright 2021 by the Turkish Osteoporosis Society / Turkish Journal of Osteoporosis published by Galenos Publishing House 


\section{Öz}

Sonuç: Postmenopozal osteoporoz hastalarında özellikle düşük serum albümin düzeyi ve/veya düşük PNI değeri lomber bölgedeki total KMY'yi olumsuz yönde etkileyebilir. Ayrıca düşük PNI değeri olan osteoporoz hastalarında Zn takviyesinin yapılması potansiyel ek bir tedavi stratejisi olabilir. Bununla birlikte Cu ve Mn bileşiklerinin takviyesi mevcut tedavilere ek olarak fayda sağlayabilir.

Anahtar kelimeler: Postmenopozal osteoporoz, prognostik nutrisyonel indeks, eser elementler

\section{Introduction}

Osteoporosis, a silent but progressive disease in which bone mineral density (BMD) and quality is affected, significantly increases the risk of fractures in postmenopausal women. As a result of the increase in the prevalence of osteoporosis with the elderly population worldwide, one in three women is at risk of osteoporotic fracture (1). The increased risk of fractures is a public health problem in postmenopausal women and also an important reason for increased morbidity and mortality. Its pathogenesis is versatile and consists of complex interactions of many physiological processes, including genetic, environmental, hormonal, and nutritional factors, as well as trace element deficiencies (2).

The presence of subclinical chronic inflammation and agerelated geriatric problems in postmenopausal osteoporosis may contribute to malnutrition development by causing deterioration of individuals' nutritional status. Therefore, postmenopausal osteoporosis and malnutrition are likely to be seen together and may affect each other negatively (3). In addition, due to both postmenopausal osteoporosis (4) and malnutrition and advanced age (5), the risk of trace element and vitamin deficiencies increases. In previous studies, it has been reported that many trace elements are required in the growth, development, and repair processes of healthy bones $(6,7)$. In some recent studies, the relationship between postmenopausal osteoporosis and trace elements such as copper (Cu), zinc (Zn), selenium (Se), and manganese (Mn) has been investigated $(8,9)$. However, although it is known that the risk of bone resorption may increase in the deficiency of $\mathrm{Cu}, \mathrm{Zn}$, Se, and Mn elements, the relationship between these elements and postmenopausal osteoporosis has not been fully elucidated $(2,10-12)$. In addition, there is no study in the literature that examines the nutritional status of postmenopausal women according to their BMD with an objective index and evaluates the relationship between trace elements. Therefore, in our study, the prognostic nutritional index (PNI), which is a reliable method that includes serum albumin and total lymphocyte count (TLC) was used for postmenopausal elderly patients (13).

In this study, we aimed to investigate whether there is a relationship between BMD and PNI and serum levels of $\mathrm{Cu}, \mathrm{Zn}$, $\mathrm{Se}$, and $\mathrm{Mn}$ trace elements in postmenopausal women.

\section{Materials and Methods}

This retrospective study included postmenopausal women who admitted to Atatürk University Medical Faculty Physical Medicine and Rehabilitation Department outpatient clinic between January 2019 and January 2020. The data of the patients included in the study were obtained from hospital records. In addition, consent was obtained from the participants. The study was approved by the Atatürk University Faculty of Medicine Ethics Committee (decision no: 16, date: 07.05.2020). The study was conducted in accordance with the Declaration of Helsinki.

In this retrospective study; 105 postmenopausal women including control ( $n=30$, T-score $>-1.0$ ); osteopenic ( $n=30,-1>$ T-score $>-2.5$ ); and osteoporotic ( $n=45, T$-score $\leq-2.5$ ) groups were investigated. Body mass index (BMI) values of women in all three groups were carefully matched.

Inclusion criteria were as follow;

1. Minimum age 45 , maximum age 70 .

2. Spontaneous menopause for at least 12 months.

Exclusion criteria were as follow;

1. Any disease history that may affect bone metabolism and cause secondary osteoporosis.

2. Having a surgical menopause.

3. Any organ pathology (i.e., gastrointestinal system) and disease that will cause malnutrition.

4. Any drug use history that may lead to corticosteroid or osteoporosis (except non-steroidal anti-inflammatory drugs and paracetamol).

5. Other medical conditions that may affect trace element levels such as kidney disease, diabetes mellitus, or medication use (i.e., diuretic, hormone replacement therapy, laxatives, etc.) may be affected.

6. Bisphosphonates, calcitonin, anabolic steroids, hormone replacement therapy, calcium, or vitamin D use in the last 6 months.

BMD measurements;

Osteoporosis diagnosis was made based on BMD measurements according to World Health Organization criteria (14). Dual-energy X-ray absorptiometry (DEXA) was evaluated by measuring the lumbar spine (L1-L4 total T-score) and femoral total and neck T-scores for axial skeletal BMD values (DEXA, Hologic QDR 2000). Group 1 (normal), group 2 (osteopenia), and group 3 (osteoporosis) were identified as bone mass loss $\leq 1$ standard deviation (SD), 1 SD< bone mass loss $<2.5$ SD, and bone mass loss $\geq 2.5 \mathrm{SD}$, respectively. Results were recorded as $\mathrm{g} / \mathrm{cm}^{2}(14)$. Determination of nutritional status;

PNI was calculated based on the equation below.

$\mathrm{PNI}=10 \times$ serum albumin $(\mathrm{g} / \mathrm{dL})+0.005 \times$ total lymphocyte count (per $\mathrm{mm}^{3}$ )

Serum albumin levels indicate protein reserve and low total lymphocyte count indicate low immune function as a result of 
malnutrition. It shows normal malnutrition if PNI value $\geq 50$, mild malnutrition if $\mathrm{PNI}$ value is $<50$, moderate to severe malnutrition if PNI value is $<45$, and severe malnutrition if PNI value is $<40$. Lower PNI scores indicate that the nutritional status is worse $(13,15)$.

Measurements of PNI parameters (albumin and TLC) in serum. Blood samples were taken from the ante-cubital vein following 8-10 hours starving and stored in standard biochemistry tube (yellow capped tubes with gel) and hemogram tubes containing ethylenediaminetetraacetic acid (EDTA). Total lymphocyte count was calculated from hemogram tubes with EDTA. Biochemistry tubes were centrifuged at 3500 rpm for 10 minutes, and serum partition was separated. Serum samples were stored at $-80^{\circ} \mathrm{C}$ until analysis. Serum samples were taken out of the refrigerator and waited until they reached room temperature. Routine clinical chemistry analyzes were carried out in clinical chemistry devices in the medical biochemistry laboratory of Atatürk University Medical Faculty. Trace element concentration measurements were performed using the Agilent ICP-MS system (Agilent technologies, Courtaboeuf, France). Normal range of serum albumin level and TLC were 3.5-5.2 g/dL and 1.16-3.61×103/ $\mathrm{mm}^{3}$, respectively.

\section{Measurements of Serum Cu, Zn, Se and Mn Levels}

The Agilent ICP-MS system consists of a 7700 Series (Agilent Technologies, Courtaboeuf, France), and the system is equipped with a third-generation octopole reaction system (ORS3) using He gas. Samples were injected from sample tubes in a CETAC ASX-500 Series autosampler (CETAC Technologies, Omaha, NE, USA) with a peristaltic pump. The gas outlet, ion lenses, solubility axis, torch position, and background are optimized daily using the adjustment solution (1 mg L-1) to perform the instrument's stability test. Normal ranges of trace elements in serum are Cu; 700-1550 mg/L, Zn; 800-1200 mg/L, Se; 63-160 mg/L and Mn; as $5-15 \mathrm{mg} / \mathrm{L}$

\section{Statistical Analysis}

The data were evaluated in the IBM SPSS statistics 25.0 (IBM Corp, Armonk, New York, USA) statistics program. Descriptive statistics are given as number of units (n), percent (\%), mean + SD ( $\mathrm{x}+\mathrm{SD})$, median (Q1-Q3) values. The normal distribution of data of numerical variables was evaluated by Shapiro-Wilk normality test and Q-Q graphs. Comparisons between groups were made using One-Way ANOVA for variables with normal distribution, and Kruskal-Wallis analysis for variables without normal distribution. As multiple comparison test, Tukey honestly significant difference was used for normally distributed variables, and Dunn-Bonferoni test was used for non-normally distributed variables. In correlations between PNI and trace elements, evaluations were made using Pearson's correlation coefficient ( $r$ ) for normally distributed variables and Spearman correlation coefficient $\left(r_{s}\right)$ for non-normally distributed variables. Linear regression analysis was performed between age, BMI, $\mathrm{PNI}$, albumin, $\mathrm{Cu}, \mathrm{Zn}$ and $\mathrm{Mn}$ variables and BMD in the groups. $\mathrm{P}<0.05$ was considered statistically significant.

\section{Results}

The average BMI, age range, and BMI values of 105 postmenopausal women were determined according to the BMD values as control ( $n=30$, mean age; $63.4 \pm 5.2$ years, age range; $53-70$ years, $\mathrm{BMI}$ mean value; $33.7 \pm 5.6$ ), osteopenia $(n=30$, mean age; $64.6 \pm 5.3$ years, age range; $50-70$ years, BMI average value; $33.5 \pm 5.9$ ) and osteoporosis ( $n=45$, average age; $65.8 \pm 5$ years, age range $51-70$ years the $\mathrm{BMI}$ average value into 30.9 \pm 7.2) groups (Table 1).

Age and BMI mean values were statistically similar in the groups. However, mean BMI values decreased gradually in the control, osteopenia, and osteoporosis groups, respectively. When PNI (albumin and TLC), serum $\mathrm{Cu}$ and $\mathrm{Zn}$ levels of the groups are compared; $\mathrm{PNI}$, serum $\mathrm{Cu}$ and $\mathrm{Zn}$ levels were found to be gradually decreased in control, osteopenia and

Table 1. Comparison of demographic characteristics, prognostic nutritional index and serum levels of trace elements in groups

\begin{tabular}{|c|c|c|c|c|}
\hline Parameters & $\begin{array}{l}\text { Control } \\
\text { (T-score: } 0 \text { to }-0.99 \text { ) }\end{array}$ & $\begin{array}{l}\text { Osteopenic } \\
\text { (T-score: }-1 \text { to }-2.49 \text { ) }\end{array}$ & $\begin{array}{l}\text { Osteoporotic } \\
\text { (T-score: } \leq-2.5 \text { ) }\end{array}$ & $\mathbf{p}$ \\
\hline$n$ & 30 & 30 & 45 & - \\
\hline Age (years) (min-max) & $63.4(53-70)$ & $64.6(50-70)$ & $65.8(51-75)$ & 0.135 \\
\hline BMI $\left(\mathrm{kg} / \mathrm{m}^{2}\right)$ & $33.7(25.5 \pm 47.6)$ & $33.5(24.4 \pm 46.8)$ & $30.9(20.1 \pm 52.3)$ & 0.109 \\
\hline PNI & $53.21+4.95$ & $52.06+4.43$ & $48.01+6.77$ & $0.005^{* *}$ \\
\hline Albumin & $42.12+2.4$ & $37.57+9.6$ & $37.96+4.5$ & $0.006^{* *}$ \\
\hline TLC & $2212+823.6$ & $2296,53+1022,1$ & $2211,11+719.3$ & 0.210 \\
\hline $\mathrm{Cu}(\mathrm{mg} / \mathrm{L})$ & $1021,4+37.4$ & $1007,2+33.5$ & $882.7+31.03$ & $0.017^{*}$ \\
\hline $\mathrm{Zn}(\mathrm{mg} / \mathrm{L})$ & $416.1+44.1$ & $366.9+40.8$ & $297.6+41.3$ & $0.026^{\star}$ \\
\hline $\mathrm{Se}(\mathrm{mg} / \mathrm{L})$ & $262.3+35.08$ & $264.2+33.5$ & $260.1+31.07$ & 0.905 \\
\hline $\mathrm{Mn}(\mathrm{mg} / \mathrm{L})$ & $9.5+2.01$ & $8.5+2.9$ & $7.4+1.9$ & 0.506 \\
\hline
\end{tabular}


osteoporosis groups, respectively. There was a statistically significant difference between control and osteoporosis groups in terms of PNI, albumin, $\mathrm{Cu}$, and $\mathrm{Zn}$ levels $(\mathrm{p}<0.05)$. However, there was no significant difference between the osteoporosis and the control groups in terms of TLC values. In addition, there was no significant difference between the osteoporosis group and the osteopenia group in terms of PNI, $\mathrm{Cu}$ and $\mathrm{Zn}$ levels, and between the osteopenia group and the control group. However, there was a significant difference between the osteopenia group and the control group in terms of albumin levels $(p<0.05)$. In control, osteopenia, and osteoporosis groups, it was observed that serum $\mathrm{Mn}$ level gradually decreased and serum Se level was lowest in the osteoporosis group, respectively. However, there was no statistically significant difference between serum $\mathrm{Mn}$ and $\mathrm{Se}$ levels between groups (Table 1).
When the relationship between the lumbar total, femur neck and femur total BMD values and demographic data, PNI and serum levels of trace elements were evaluated; there was a weak negative correlation between age and femoral neck BMD In the control and osteoporosis groups and a moderate positive correlation between BMI and femoral neck and femur total BMD values in the osteopenia group $(p<0.05)$. There was a moderate $(p=0.027, r=0.402)$ and weakly positive correlation $(p=0.023$, $r=0.340$ ) between the lumbar total BMD values and PNI in the control and osteoporosis groups, respectively. In addition, there was a low $(p=0.032, r=0.393)$ and moderate $(p=0.001$, $r=0.556$ ) positive correlation between femur neck and femur total BMD values and albumin in the control group. There was a weak positive correlation between the lumbar total BMD and albumin in the osteoporosis group ( $p=0.008, r=0.392$ ) (Table 2). There was a moderate positive correlation between femur neck

Table 2. Relationship between bone mineral density and demographic characteristics, prognostic nutritional index and serum levels of trace elements in groups

\begin{tabular}{|c|c|c|c|c|c|c|c|c|c|c|c|c|c|c|c|c|c|c|}
\hline & \multicolumn{6}{|c|}{ Control } & \multicolumn{6}{|c|}{ Osteopeni } & \multicolumn{6}{|c|}{ Osteoporosis } \\
\hline & \multirow{2}{*}{\multicolumn{2}{|c|}{$\begin{array}{l}\text { Lomber } \\
\text { total } \\
\text { BMD } \\
\left(\mathrm{g} / \mathrm{cm}^{2}\right)\end{array}$}} & \multirow{2}{*}{\multicolumn{2}{|c|}{$\begin{array}{l}\text { Femur } \\
\text { neck } \\
\text { BMD } \\
\left(\mathrm{g} / \mathrm{cm}^{2}\right)\end{array}$}} & \multirow{2}{*}{\multicolumn{2}{|c|}{$\begin{array}{l}\text { Femur } \\
\text { total } \\
\text { BMD } \\
\left(\mathrm{g} / \mathrm{cm}^{2}\right)\end{array}$}} & \multirow{2}{*}{\multicolumn{2}{|c|}{$\begin{array}{l}\text { Lomber } \\
\text { total } \\
\text { BMD } \\
\left(\mathrm{g} / \mathrm{cm}^{2}\right)\end{array}$}} & \multirow{2}{*}{\multicolumn{2}{|c|}{$\begin{array}{l}\text { Femur } \\
\text { neck } \\
\text { BMD } \\
\left(\mathrm{g} / \mathrm{cm}^{2}\right)\end{array}$}} & \multirow{2}{*}{\multicolumn{2}{|c|}{$\begin{array}{l}\text { Femur } \\
\text { total } \\
\text { BMD } \\
\left(\mathrm{g} / \mathrm{cm}^{2}\right)\end{array}$}} & \multirow{2}{*}{\multicolumn{2}{|c|}{$\begin{array}{l}\text { Lomber } \\
\text { total } \\
\text { BMD } \\
\left(\mathrm{g} / \mathrm{cm}^{2}\right)\end{array}$}} & \multirow{2}{*}{\multicolumn{2}{|c|}{$\begin{array}{l}\text { Femur } \\
\text { neck } \\
\text { BMD } \\
\left(\mathrm{g} / \mathrm{cm}^{2}\right)\end{array}$}} & \multirow{2}{*}{\multicolumn{2}{|c|}{$\begin{array}{l}\begin{array}{l}\text { Femur } \\
\text { total } \\
\text { BMD }\end{array} \\
\left(\mathrm{g} / \mathrm{cm}^{2}\right)\end{array}$}} \\
\hline & & & & & & & & & & & & & & & & & & \\
\hline & $\mathbf{p}$ & $r$ & p & $r$ & $\mathbf{p}$ & $\mathbf{r}$ & $p$ & $r$ & $p$ & r & $p$ & $r$ & $p$ & $r$ & $\mathbf{p}$ & $r$ & $\mathbf{p}$ & $r$ \\
\hline Age & $\begin{array}{l}\infty \\
\underset{N}{0} \\
0\end{array}$ & $\underset{ָ}{\approx}$ & $\begin{array}{l}\stackrel{*}{\circ} \\
\text { Ð } \\
\stackrel{0}{\circ}\end{array}$ & 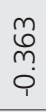 & 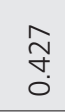 & $\begin{array}{l}\text { Е } \\
\stackrel{0}{i}\end{array}$ & $\begin{array}{l}\infty \\
0 \\
\text { m. } \\
0\end{array}$ & 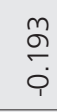 & $\begin{array}{l}\text { J } \\
0 \\
0\end{array}$ & $\begin{array}{l}\text { o } \\
\text { Oे } \\
\text { i }\end{array}$ & $\begin{array}{l}\text { ণ্ } \\
\text { o. }\end{array}$ & $\begin{array}{l}\bar{m} \\
\stackrel{0}{i}\end{array}$ & $\begin{array}{l}\text { Ln } \\
\infty \\
0 \\
0\end{array}$ & $\begin{array}{l}\infty \\
m \\
i\end{array}$ & $\begin{array}{l}\text { * } \\
\text { ○ } \\
0 \\
0\end{array}$ & 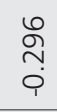 & $\frac{N}{0}$ & 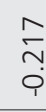 \\
\hline BMI & $\begin{array}{l}\infty \\
\stackrel{\infty}{n} \\
0\end{array}$ & 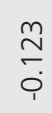 & 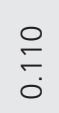 & $\begin{array}{l}\infty \\
\stackrel{0}{0} \\
\text { ○ }\end{array}$ & $\begin{array}{l}\frac{0}{\sigma} \\
0\end{array}$ & $\begin{array}{l}\hat{\text { స్ }} \\
\text { ọ }\end{array}$ & $\begin{array}{l}\infty \\
\sigma \\
0 \\
0\end{array}$ & $\begin{array}{l}\text { ஸी } \\
\text { о } \\
\text { 우 }\end{array}$ & $\begin{array}{l}\stackrel{*}{\circ} \\
\stackrel{0}{\circ} \\
\circ\end{array}$ & \begin{tabular}{l}
$n$ \\
6 \\
\multirow{0}{*}{} \\
0
\end{tabular} & 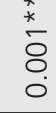 & $\begin{array}{l}\stackrel{n}{N} \\
\text { ஸी } \\
0\end{array}$ & 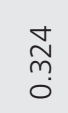 & 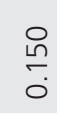 & $\begin{array}{l}\infty \\
\text { ᄋ̆ } \\
\text { ? }\end{array}$ & $\stackrel{\infty}{\oplus}$ & $\begin{array}{l}\text { ㅇ } \\
\text { ○े } \\
0\end{array}$ & 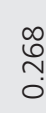 \\
\hline PNI & $\begin{array}{l}\stackrel{*}{\curvearrowright} \\
\text { ○ } \\
0 \\
0\end{array}$ & $\begin{array}{l}\text { Oे } \\
\text { +े } \\
\text { ○ }\end{array}$ & $\begin{array}{l}\infty \\
0 \\
0 \\
0\end{array}$ & $\begin{array}{l}0 \\
\stackrel{1}{\circ} \\
\circ \\
0\end{array}$ & 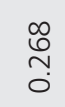 & $\begin{array}{l}\text { ᄋ } \\
\text { ஸे } \\
0\end{array}$ & $\begin{array}{l}\text { ปิ } \\
8 \\
0\end{array}$ & 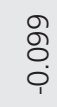 & $\begin{array}{l}\stackrel{n}{N} \\
\vdots \\
0\end{array}$ & $\begin{array}{l}\text { Ðె } \\
\varnothing \\
0\end{array}$ & $\frac{\Sigma}{\tilde{0}}$ & 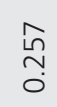 & $\begin{array}{l}\stackrel{*}{n} \\
\stackrel{\sim}{O} \\
0\end{array}$ & $\begin{array}{l}\stackrel{+}{+} \\
\text { mo } \\
\text { o }\end{array}$ & $\stackrel{\circ}{m}$ & $\frac{\stackrel{\llcorner}{\Perp}}{\stackrel{0}{0}}$ & $\begin{array}{l}\text { ڤ్ } \\
\stackrel{0}{0}\end{array}$ & $\begin{array}{l}\text { 음 } \\
\text { ○. }\end{array}$ \\
\hline Alb & $\begin{array}{l}N \\
\infty \\
0 \\
0\end{array}$ & 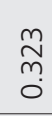 & $\begin{array}{l}\stackrel{*}{\cong} \\
\stackrel{0}{O} \\
\stackrel{0}{0}\end{array}$ & 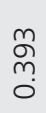 & $\stackrel{*}{*} 8$ & $\begin{array}{l}0 \\
\text { ผก } \\
\text { ก? } \\
0\end{array}$ & $\begin{array}{l}\hat{0} \\
\dot{0} \\
0\end{array}$ & $\begin{array}{l}\infty \\
\text { ○ } \\
\circ \\
0\end{array}$ & $\begin{array}{l}\infty \\
\infty \\
\stackrel{\infty}{ก} \\
0\end{array}$ & $\frac{n}{\stackrel{0}{0}}$ & 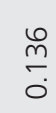 & $\underset{\substack{\infty \\
\hdashline}}{\stackrel{\infty}{0}}$ & $\begin{array}{l}* \\
* \\
\infty \\
\stackrel{0}{8} \\
0\end{array}$ & $\underset{\mathrm{N}}{\mathrm{N}}$ & 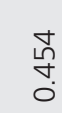 & $\frac{\Xi}{\check{\sigma}}$ & $\begin{array}{l}\stackrel{n}{\Upsilon} \\
\stackrel{0}{0} \\
0\end{array}$ & 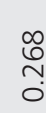 \\
\hline TLC & $\begin{array}{l}\frac{6}{E} \\
0\end{array}$ & $\begin{array}{l}\text { ñ } \\
\text { No }\end{array}$ & $\begin{array}{l}8 \\
\stackrel{+}{0} \\
0\end{array}$ & $\begin{array}{l}\stackrel{\circ}{n} \\
\dot{0}\end{array}$ & $\begin{array}{l}\infty \\
\stackrel{0}{0} \\
0 \\
0\end{array}$ & $\begin{array}{l}\overline{\hat{O}} \\
\text { o }\end{array}$ & 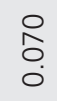 & $\begin{array}{l}\stackrel{\operatorname{m}}{m} \\
m \\
0\end{array}$ & 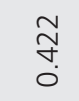 & $\frac{N}{\stackrel{\text { กิ }}{\circ}}$ & 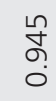 & $\begin{array}{l}m \\
\vdots \\
0\end{array}$ & $\begin{array}{l}\stackrel{+}{m} \\
m \\
0\end{array}$ & $\frac{\tilde{\sigma}}{\circ}$ & $\begin{array}{l}\stackrel{m}{m} \\
m \\
0\end{array}$ & 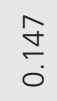 & $\frac{0}{\stackrel{0}{\circ}}$ & 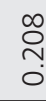 \\
\hline $\mathrm{Cu}$ (mg/L) & $\frac{\stackrel{\infty}{+}}{\circ}$ & \begin{tabular}{l}
$\bar{N}$ \\
\multirow{1}{*}{} \\
0
\end{tabular} & $\begin{array}{l}\stackrel{*}{*} \\
\stackrel{*}{8} \\
0\end{array}$ & 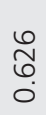 & $\frac{*}{\tilde{\sigma}}$ & 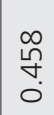 & $\begin{array}{l}\text { ठ } \\
\text { Ø̣ } \\
0\end{array}$ & $\stackrel{m}{N}$ & $\begin{array}{l}m \\
\infty \\
\infty \\
0\end{array}$ & 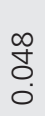 & $\begin{array}{l}\bar{N} \\
\infty \\
0\end{array}$ & $\begin{array}{l}\bar{m} \\
0 \\
0\end{array}$ & $\begin{array}{l}\Omega \\
6 \\
0 \\
0\end{array}$ & $\begin{array}{l}\text { ñ } \\
\stackrel{0}{0} \\
0\end{array}$ & $\stackrel{\text { N }}{\underset{0}{0}}$ & $\frac{8}{8}$ & $\begin{array}{l}\infty \\
\text { న్ } \\
0\end{array}$ & $\begin{array}{l}\overline{8} \\
\circ \\
\circ\end{array}$ \\
\hline $\mathrm{Zn}(\mathrm{mg} / \mathrm{L})$ & 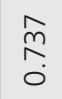 & $\begin{array}{l}\text { ఫे } \\
\text { ర़ } \\
0\end{array}$ & $\begin{array}{l}\infty \\
\stackrel{\infty}{n} \\
0 \\
0\end{array}$ & \begin{tabular}{l} 
○ \\
\multirow{+}{0}{} \\
0
\end{tabular} & 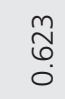 & $\begin{array}{l}0 \\
\text { กำ } \\
0\end{array}$ & $\begin{array}{l}m \\
m \\
\infty \\
0\end{array}$ & 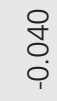 & $\stackrel{\frac{m}{\pi}}{\stackrel{0}{0}}$ & $\begin{array}{l}\stackrel{0}{\circ} \\
\text { O } \\
0\end{array}$ & $\begin{array}{l}\overline{0} \\
0 \\
0\end{array}$ & $\frac{8}{\circ}$ & $\underset{\infty}{\infty}$ & $\underset{⿱ 亠}{\stackrel{+}{0}}$ & $\begin{array}{l}\bar{\alpha} \\
\infty \\
0\end{array}$ & 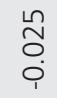 & $\begin{array}{l}\text { ○ } \\
\text { రి } \\
\dot{0}\end{array}$ & $\begin{array}{l}\text { ㅇ } \\
\text { 웅 } \\
0\end{array}$ \\
\hline $\mathrm{Se}(\mathrm{mg} / \mathrm{L})$ & $\begin{array}{l}\infty \\
\varnothing \\
\varnothing \\
0\end{array}$ & $\begin{array}{l}\circ \\
\infty \\
m \\
0 \\
0\end{array}$ & $\begin{array}{l}\text { ळે } \\
\infty \\
0 \\
0\end{array}$ & $\begin{array}{l}\stackrel{+}{\sim} \\
\text { O̦ } \\
0\end{array}$ & $\begin{array}{l}\bar{\sigma} \\
0 \\
0\end{array}$ & $\begin{array}{l}\stackrel{\circ}{గ} \\
\text { ○ } \\
0\end{array}$ & \begin{tabular}{l}
$m$ \\
$\infty$ \\
\multirow{0}{0}{}
\end{tabular} & $\begin{array}{l}\text { N̂} \\
\text { O } \\
0\end{array}$ & 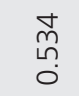 & $\frac{\infty}{\check{\sigma}}$ & 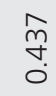 & $\begin{array}{l}\text { 于 } \\
\stackrel{1}{0}\end{array}$ & $\begin{array}{l}8 \\
80 \\
0 \\
0\end{array}$ & 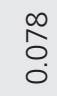 & 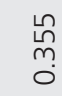 & $\frac{\bar{J}}{\dot{0}}$ & $\underset{\stackrel{\sim}{\sim}}{\stackrel{\sim}{\circ}}$ & 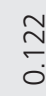 \\
\hline $\mathrm{Mn}(\mathrm{mg} / \mathrm{L})$ & 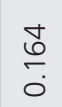 & $\begin{array}{l}\bar{\sigma} \\
\stackrel{1}{0} \\
0\end{array}$ & $\begin{array}{l}8 \\
\circ \\
\circ \\
0\end{array}$ & $\stackrel{n}{m}$ & 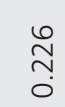 & $\stackrel{\infty}{\underset{N}{N}}$ & స్ & $\begin{array}{l}\text { m } \\
\text { ○ } \\
0\end{array}$ & $\begin{array}{l}\hat{n} \\
m \\
0\end{array}$ & $\frac{⿱}{\check{0}}$ & $\stackrel{\text { ণ }}{\stackrel{\leftarrow}{\circ}}$ & $\stackrel{\circ}{\stackrel{\circ}{\circ}}$ & $\begin{array}{l}\stackrel{*}{m} \\
\stackrel{0}{0} \\
0\end{array}$ & $\underset{\sim}{\mathbb{N}}$ & $\underset{\text { I }}{\stackrel{\text { m}}{\circ}}$ & $\frac{\text { O }}{\stackrel{+}{+}}$ & $\stackrel{\stackrel{0}{\circledR}}{\stackrel{\leftrightarrow}{\circ}}$ & $\begin{array}{l}\text { ণ } \\
\text { : } \\
\text { Q }\end{array}$ \\
\hline
\end{tabular}


and femur total BMD values and serum Cu levels in the control group (respectively; $p=0.001, r=0.626$ and $p=0.011, r=0.455$ ) while a weak positive correlation was observed between lumbar total $B M D$ values and serum $\mathrm{Mn}(p=0.031, r=0.32)$ levels in the osteoporosis group $(p<0.05)$. However, there was no correlation between serum $\mathrm{Zn}$ and Se values and BMD values (Table 2). When the relationships between PNI values and demographic data and serum levels of trace elements are evaluated; there was a significant positive moderate correlation between PNI level and serum $\mathrm{Zn}$ level ( $\mathrm{p}=0.045, \mathrm{r}=0.063$ ) in osteoporosis group, but there was no significant correlation in other data (Table 3).

Age, BMI, PNI, albumin, $\mathrm{Cu}, \mathrm{Mn}$ and $\mathrm{Zn}$ levels were included in the linear regression analysis to reveal the role of the analyzed parameters in the determination of BMD. It was observed that PNI value is not a determiner on lumbar total BMD in postmenopausal osteoporosis patients while the albumin level is a weak determinant [Beta coefficient $(\beta)=0.556, R^{2}=0.309$, $p=0.001$, standard error $=0.054,95 \%$ confidence interval] (Graphic 1). It was observed that BMI was a weak determinant on femur neck and femur total BMD in osteopenia patients while PNI value was found to be a weak determinant on lumbar total BMD in control patients. It was observed that the other parameters evaluated were not a determining factor on BMD.

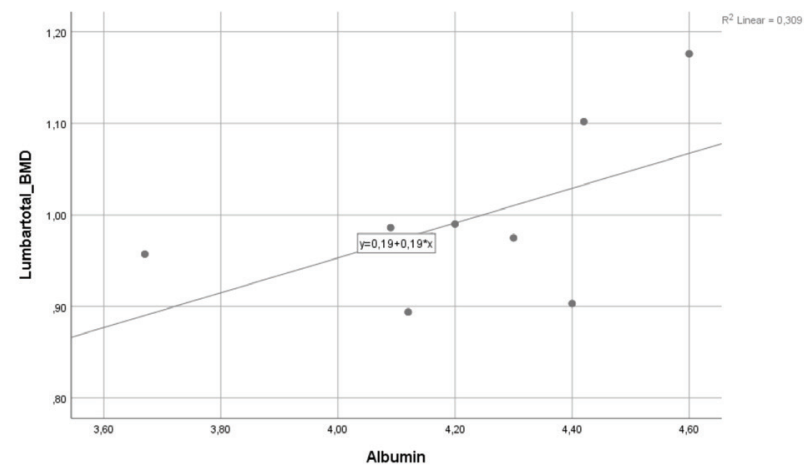

Graphic 1. Linear regression graph between albumin and lumbar total BMD in postmenopausal osteoporosis group $B M D$ : Body mineral density

\section{Discussion}

In our study, it was found that PNI, serum albumin, $\mathrm{Cu}$, and $\mathrm{Zn}$ levels were significantly lower in the osteoporosis group compared to the control group, and there was a weak positive correlation between lumbar total BMD values and PNI, albumin, and serum Mn levels. In addition, there was a moderate positive correlation between $\mathrm{PNI}$ and serum Zn levels in the osteoporosis group.

Osteoporosis, one of the most important health problems of postmenopausal women and genetic factors play a major role. However, adequate and balanced nutrition can be an inexpensive and easily applicable therapeutic method as well as an additional contribution to the treatment process compared to other treatment methods. Trace elements other than vitamin D and calcium, which are known as the main components of bone metabolism are essential sub-elements necessary for the success of many stages of bone metabolism (16). For this reason, deficiencies of these basic sub-items can be eliminated with adequate and balanced nutrition or supplements in postmenopausal osteoporosis. Otherwise, in addition to the increased morbidity and mortality seen in osteoporosis, additional morbidity and mortality in malnutrition may pose a serious risk (17).

While advanced age and low BMI values contribute to the formation of osteoporosis in postmenopausal women, subclinical chronic inflammation present in osteoporosis patients may also contribute to the formation of malnutrition (17-20). In our study, there was no significant difference between the groups in terms of age and BMI values. Therefore, it can be suggested that age and BMI values in the groups did not make an additional contribution to the formation of postmenopausal osteoporosis and malnutrition. However, similar to the literature, postmenopausal patients had the lowest BMI level, and this may be an indicator of impaired nutritional status in patients with osteoporosis. Furthermore, it was found that there was a significant relationship between BMI and femur neck and femur total BMD in osteopenia patients. The regression analysis revealed that $\mathrm{BMI}$ had a strong effect on femur neck and femur total BMD.

The albumin level evaluated in the PNI is an important indicator of the amount of protein taken by the diet (21). There are several

Table 3. Relationship between prognostic nutritional index values and serum levels of trace elements in groups

\begin{tabular}{|c|c|c|c|c|c|c|}
\hline \multirow[t]{2}{*}{ Parameters } & \multicolumn{2}{|c|}{$\begin{array}{l}\text { PNI values with normal control } \\
\text { (T-score: } 0 \text { to }-0.99 \text { ) }\end{array}$} & \multicolumn{2}{|c|}{$\begin{array}{l}\text { PNI values with osteopenic } \\
\text { (T-score: }-1 \text { to }-2.49 \text { ) }\end{array}$} & \multicolumn{2}{|c|}{$\begin{array}{l}\text { PNI values with osteoporotic } \\
\text { (T-score: } \leq-2.5)\end{array}$} \\
\hline & $\mathbf{p}$ & $r$ & p & $r$ & $\mathbf{p}$ & $r$ \\
\hline Age (years) & 0.913 & -0.21 & 0.098 & -0.308 & 0.891 & -0.21 \\
\hline $\mathrm{BMI}\left(\mathrm{kg} / \mathrm{m}^{2}\right)$ & 0.256 & 0.214 & 0.394 & 0.162 & 0.868 & -0.025 \\
\hline $\mathrm{Cu}(\mathrm{mg} / \mathrm{L})$ & 0.052 & -0.359 & 0.946 & 0.013 & 0.605 & -0.079 \\
\hline $\mathrm{Zn}(\mathrm{mg} / \mathrm{L})$ & 0.671 & -0.081 & 0.181 & -0.251 & $0.045^{\star}$ & 0.063 \\
\hline $\mathrm{Se}(\mathrm{mg} / \mathrm{L})$ & 0.104 & 0.302 & 0.552 & -0.113 & 0.761 & -0.047 \\
\hline $\mathrm{Mn}(\mathrm{mg} / \mathrm{L})$ & 0.909 & -0.022 & 0.717 & 0.069 & 0.329 & 0.149 \\
\hline
\end{tabular}


epidemiological studies reporting that there is a relationship between dietary protein and bone $(22,23)$. These studies show that individuals who consume the most dietary protein have the highest BMD $(24,25)$. However, there are studies reporting that there is no independent relationship between low albumin levels and BMD $(26,27)$. TLC is a useful determinant in the determination of nutritional status, and it is a fast, easily accessible method that can be used in all age groups $(13,15,28)$. In addition, it has been stated that TLC may be a suitable marker in the evaluation of nutritional status and bone health together with albumin in advanced age (senile) patients (29). However, although low TLC is not a specific finding for malnutrition, it has been reported that it may show decreased immune function resulting from malnutrition $(13,15,28)$.

Although the mechanism of the relationship between postmenopausal osteoporosis and hypoalbuminemia is not clear; hypoalbuminemia may be directly related to nuclear factor kappa B, an important protein complex known to suppress osteogenesis and osteoclast activation. Or indirect interaction with acute phase reactants, such as TNF-alpha, IL-1, can also cause a decrease in BMD. Hypoalbuminemia may cause decreased BMD in individuals affected by diseases associated with low albumin levels or through various mechanisms caused by the deficiency of many proteins and trace elements carried by albumin (30). In our study, when the PNI values in the groups were examined, it was seen that the nutritional status was normal in the control and osteopenia groups, but mild malnutrition was observed in the osteoporosis group. PNI and albumin levels were significantly lower in patients with postmenopausal osteoporosis than in control patients. In addition, a positive correlation was found between lumbar total BMD value and PNI and albumin levels in postmenopausal osteoporosis patients. However, the regression analysis revealed that albumin level had a weak effect on lumbar total BMD. Therefore, our study was found to be compatible with previous literature information suggesting a relationship between albumin deficiency and osteoporosis (30). However, it can show us that albumin deficiency may have an important effect on especially trabecular bone density and BMD decrease in lumbar vertebrae where metabolic activities are high, and some precautions should be taken in terms of nutritional status and bone health. In our study, it was found that there was no significant difference between the TLC levels of the groups, and there was no relationship between BMD and TLC levels. This may be due to the very close PNI values in the groups, the limited number of patients, and other factors that may affect the TLC.

The relationship between $\mathrm{Cu}, \mathrm{Zn}, \mathrm{Se}$, and $\mathrm{Mn}$ and osteoporosis is not well defined in the literature. Although there are studies indicating that these trace elements decrease in most patients with postmenopausal osteoporosis, there are studies reporting the opposite $(2,8,10-12)$. Therefore, we determined the serum levels of $\mathrm{Cu}, \mathrm{Zn}, \mathrm{Se}$, and $\mathrm{Mn}$ trace elements and focused on the relationship between $\mathrm{BMD}$ and $\mathrm{PNI}$.
Although the $\mathrm{Cu}$ deficiency is rarely reported in humans, a low or moderate $\mathrm{Cu}$ deficiency may affect the synthesis and stability of bone collagen or cause osteogenesis inhibition leading to osteoporosis $(31,32)$. This indicates that adequate $\mathrm{Cu}$ intake with diet is important for the preservation of bone and cartilage (33). However, there are studies reporting that there is no difference between serum Cu levels of patients with and without postmenopausal osteoporosis (2). In our study, serum $\mathrm{Cu}$ levels in the groups were within the normal range. However, the lowest $\mathrm{Cu}$ level was found in patients with postmenopausal osteoporosis, and there was a significant difference between the control group. Therefore, it suggests that it may be a new supplement candidate in the prevention and treatment of osteoporosis. Although there was no relationship between BMD and serum $\mathrm{Cu}$ level in osteoporosis and osteopenia patients, a significant positive relationship was found in the control group. This may have occurred due to other factors that may affect the serum Cu level in the groups. There was no relationship between serum Cu level and PNI in our study. This may be due to the fact that serum $\mathrm{Cu}$ is less affected by the albumin level because more than $90 \%$ of serum $\mathrm{Cu}$ is transported via ceruloplasmin. In addition, since serum Cu level is within the normal range in groups, it can be thought that hematopoiesis and/or TLC are not affected by this condition.

$\mathrm{Zn}$ is being the cofactor of enzymes such as alkaline phosphatase, collagenase, and sulfurethylase, which are involved in the synthesis of various bone matrix components and have important roles in normal collagen synthesis and bone mineralization. There are many studies reporting that there is a relationship between $\mathrm{Zn}$ deficiency and osteoporosis $(2,8,9,11,12)$.

In our study, serum Zn levels were found to be below normal levels in all groups. In addition, it was determined that the lowest Zn level was in postmenopausal osteoporosis patients, and there was a significant difference between the other groups. In addition, although there was no relationship between BMD and serum Zn level, a positive relationship was found between PNI and $\mathrm{Zn}$ level in patients with osteoporosis. The fact that albumin acts as the basic $\mathrm{Zn}$-binding protein in plasma may be the reason for the positive relationship between PNI and Zn in our study. This may show us that in patients with osteoporosis with a low PNI value, Zn supplementation may be appropriate in addition to their current treatment and maybe a new potential additional treatment in the prevention and treatment of osteoporosis.

Although there are many studies evaluating the relationship between Se and BMD in the current literature, the results are insufficient (34). While Se level was positively associated with BMD in healthy aging euthyroid postmenopausal women (35), another study conducted on postmenopausal 50-79-year-old participants suggested that dietary Se intake was not associated with BMD (36). In our study, although there was no difference between serum Se levels in the groups, the lowest Se level was found to be in the osteoporosis group. In addition, no correlation was found between serum Se levels and BMD and PNI. Similar to our study, other studies evaluating the relationship between 
Se and BMD in the literature evaluated serum Se levels rather than the amount of Se intake in the diet. This may be a limitation in the context of examining the relationship with Se. Therefore, we think that further studies are needed on the subject.

A large part of the $\mathrm{Mn}$ in the body is in the bone, and the $\mathrm{Mn}$ density of the bone is an indicator of the serum $\mathrm{Mn}$ level and diet Mn, just like Cu and Zn (37). In a study, it was reported that the $\mathrm{Mn}$ level in serum was lower in postmenopausal women with osteoporosis compared to non-osteoporotic controls (38). In a study conducted by Strause et al. (39), it was reported that replacement of $\mathrm{Mn}$ together with $\mathrm{Zn}$ and $\mathrm{Cu}$ might help to reduce lumbar region BMD loss in postmenopausal women. In our study, serum Mn levels in the groups were within the normal range. The narrow range of serum $\mathrm{Mn}$ level and rareness of deficiency is confirmed by our findings. In our study, the lowest Mn level was found in postmenopausal osteoporosis patients, and there was a weak positive relationship between lumbar total BMD and serum Mn level. While this supports the study of Strause et al. (39), it suggests that Mn compounds may be a new supplement candidate in the prevention and treatment of osteoporosis. However, the lack of relationship between PNI and serum $\mathrm{Mn}$ level in our study may be due to the limited number of patients or especially the role beta globulin in addition to albumin in the transport of $\mathrm{Mn}$ to bone.

\section{Study Limitations}

Limitations of our study can be listed as the limited number of patients, lack of information about how much of the trace elements are taken with the diet, the evaluation of only serum levels of trace elements and the effects of many factors on serum levels of trace elements such as hormone replacement therapy, diuretic and laxative use.

\section{Conclusion}

Especially low serum albümin levels and low PNI value in postmenopausal osteoporosis patients may adversely affect total BMD in the lumbar region. Additionally, supplementing $\mathrm{Zn}$ in osteoporosis patients with low PNI can be a potential additional treatment strategy. However, supplementation of the $\mathrm{Cu}$ and $\mathrm{Mn}$ compounds may provide an additional benefit to existing treatments. However, we think that our findings should be supported with more comprehensive future studies investigating the nutritional aspect of osteoporosis and since the question of which supplements should be used is very complex, and the mechanisms are not yet fully understood.

\section{Ethics}

Ethics Committee Approval: The study was approved by the Atatürk University Faculty of Medicine Ethics Committee (decision no: 16, date: 07.05.2020).

Informed Consent: In addition, consent was obtained from the participants.

Peer-review: Externally and internally peer-reviewed.

\section{Authorship Contributions}

Surgical and Medical Practices: A.K, Z.B, M.Ç., Concept: A.K, Z.B, M.Ç., Design: A.K, Z.B, M.Ç., Data Collection or Processing: A.K, Z.B, M.Ç., Analysis or Interpretation: A.K, Z.B, Literature Search: A.K., Z.B, Writing: A.K., Z.B.

Conflict of Interest: No conflict of interest was declared by the authors.

Financial Disclosure: The authors declared that this study has received no financial support.

\section{References}

1. Higgs J, Derbyshire E, Styles K. Nutrition and osteoporosis prevention for the orthopaedic surgeon: A wholefoods approach. EFORT Open Rev 2017;2:300-8.

2. Arikan DC, Coskun A, Ozer A, Kilinc M, Atalay F, Arikan T. Plasma selenium, zinc, copper and lipid levels in postmenopausal Turkish women and their relation with osteoporosis. Biol Trace Elem Res 2011;144:407-17.

3. Montalcini T, Romeo S, Ferro Y, Migliaccio V, Gazzaruso C, Pujia A. Osteoporosis in chronic inflammatory disease: the role of malnutrition. Endocrine 2013;43:59-64.

4. Saltman PD, Strause LG. The role of trace minerals in osteoporosis. J Am Coll Nutr 1993;12:384-9.

5. Bonaccorsi G, Lorini C, Bani Assad G, Pepe P, Santomauro F. Serum trace elements and risk of malnutrition in institutionalised elderly. Eur J Clin Nutr 2013;67:155-60.

6. Saltman P, Strause L. Trace elements in bone metabolism and osteoporosis. J Inorg Biochem 1991;43:284.

7. Saltman PD, Strause LG. The role of trace minerals in osteoporosis. J Am Coll Nutr 1993;12:384-9.

8. Odabasi E, Turan M, Aydin A, Akay C, Kutlu M. Magnesium, zinc, copper, manganese, and selenium levels in postmenopausal women with osteoporosis. Can magnesium play a key role in osteoporosis? Ann Acad Med Singap 2008;37:564-7.

9. Liu SZ, Yan H, Xu P, Li JP, Zhuang GH, Zhu BF, et al. Correlation analysis between bone mineral density and serum element contents of postmenopausal women in Xi'an urban area. Biol Trace Elem Res 2009;131:205-14.

10. Wang L, Yu H, Yang G, Zhang Y, Wang W, Su T, et al. Correlation between bone mineral density and serum trace element contents of elderly males in Beijing urban area. Int J Clin Exp Med 2015;8:19250-7.

11. Strain JJ. A reassessment of diet and osteoporosis-possible role for copper. Med Hypotheses 1988;27:333-8.

12. Rodríguez J, Mandalunis PM. A Review of Metal Exposure and Its Effects on Bone Health. J Toxicol 2018;2018:4854152.

13. Sattler ELP, Ishikawa $Y$, Trivedi-Kapoor R, Zhang D, Quyyumi AA, Dunbar SB. Association between the Prognostic Nutritional Index and Dietary Intake in Community-Dwelling Older Adults with Heart Failure: Findings from NHANES III. Nutrients 2019;11:2608.

14. Miller PD. Guidelines for the diagnosis of osteoporosis: T-scores vs fractures. Rev Endocr Metab Disord 2006;7:75-89.

15. Benjamin EJ, Virani SS, Callaway CW, Chamberlain AM, Chang AR, Cheng S, et al. Heart Disease and Stroke Statistics-2018 Update: A Report From the American Heart Association. Circulation 2018;137:67-492.

16. Coxam V, Wauquier F, Darie C, Spilmont M, Davicco MJ, Wittrant Y. Huile d'olive et santé osseuse. OCL 2014;21:D5111.

17. Lee LC, Tsai AC, Wang JY, Hurng BS, Hsu HC, Tsai HJ. Need-based intervention is an effective strategy for improving the nutritional status of older people living in a nursing home: a randomized controlled trial. Int J Nurs Stud 2013;50:1580-8.

18. Dargent-Molina P, Poitiers F, Bréart G; EPIDOS Group. In elderly women weight is the best predictor of a very low bone mineral density: evidence from the EPIDOS study. Osteoporos Int 2000;11:881-8. 
19. Lacativa PG, Farias ML. Osteoporosis and inflammation. Arq Bras Endocrinol Metabol 2010;54:123-32.

20. Montalcini T, Romeo S, Ferro Y, Migliaccio V, Gazzaruso C, Pujia A. Osteoporosis in chronic inflammatory disease: the role of malnutrition. Endocrine 2013;43:59-64.

21. Peters BS, Martini LA. Nutritional aspects of the prevention and treatment of osteoporosis. Arq Bras Endocrinol Metabol 2010;54:179-85.

22. Henriques R, Jásik J, Klein M, Martinoia E, Feller U, Schell J, et al. Knock-out of Arabidopsis metal transporter gene IRT1 results in iron deficiency accompanied by cell differentiation defects. Plant Mol Biol 2002;50:587-97.

23. Ryu MS, Lichten LA, Liuzzi JP, Cousins RJ. Zinc transporters ZnT1 (Slc30a1), Zip8 (SIc39a8), and Zip10 (Slc39a10) in mouse red blood cells are differentially regulated during erythroid development and by dietary zinc deficiency. J Nutr 2008;138:2076-83.

24. Kanno $S$, Anuradha CD, Hirano S. Localization of zinc after in vitro mineralization in osteoblastic cells. Biol Trace Elem Res 2001;83:3947.

25. Lopez V, Kelleher SL. Zinc transporter-2 (ZnT2) variants are localized to distinct subcellular compartments and functionally transport zinc. Biochem J 2009;422:43-52.

26. D'Erasmo E, Pisani D, Ragno A, Raejntroph N, Letizia C, Acca M. Relationship between serum albumin and bone mineral density in postmenopausal women and in patients with hypoalbuminemia. Horm Metab Res 1999;31:385-8.

27. Lunde AV, Barrett-Connor E, Morton DJ. Serum albumin and bone mineral density in healthy older men and women: the Rancho Bernardo Study. Osteoporos Int 1998;8:547-51.

28. Nishida T, Sakakibara H. Association between underweight and low lymphocyte count as an indicator of malnutrition in Japanese women. J Womens Health (Larchmt) 2010;19:1377-83.

29. Di Monaco M, Vallero F, Di Monaco R, Mautino F, Cavanna A. Biochemical markers of nutrition and bone mineral density in the elderly. Gerontology 2003;49:50-4.
30. Afshinnia F, Wong KK, Sundaram B, Ackermann RJ, Pennathur S. Hypoalbuminemia and Osteoporosis: Reappraisal of a Controversy. J Clin Endocrinol Metab 2016;101:167-75.

31. Arredondo M, Núñez MT. Iron and copper metabolism. Mol Aspects Med 2005;26:313-27.

32. Rodríguez JP, Ríos S, González M. Modulation of the proliferation and differentiation of human mesenchymal stem cells by copper. J Cell Biochem 2002;85:92-100.

33. Aaseth J, Boivin G, Andersen O. Osteoporosis and trace elementsan overview. J Trace Elem Med Biol 2012;26:149-52.

34. Wang Y, Xie D, Li J, Long H, Wu J, Wu Z, et al. Association between dietary selenium intake and the prevalence of osteoporosis: a cross-sectional study. BMC Musculoskelet Disord 2019;20:585.

35. Beukhof CM, Medici M, van den Beld AW, Hollenbach B, Hoeg A, Visser WE, et al. Selenium Status Is Positively Associated with Bone Mineral Density in Healthy Aging European Men. PLoS One 2016;11:e0152748.

36. Wolf RL, Cauley JA, Pettinger M, Jackson R, Lacroix A, Leboff MS, et al. Lack of a relation between vitamin and mineral antioxidants and bone mineral density: results from the Women's Health Initiative. Am J Clin Nutr 2005;82:581-8.

37. Dermience M, Lognay G, Mathieu F, Goyens P. Effects of thirty elements on bone metabolism. J Trace Elem Med Biol 2015;32:86106.

38. Reginster JY, Strause L, Deroisy R, Lecart MP, Saltman $P_{\text {, }}$ Franchimont P. Preliminary report of decreased serum magnesium in postmenopausal osteoporosis. Magnesium 1989;8:106-9.

39. Strause L, Saltman P, Smith KT, Bracker M, Andon MB. Spinal bone loss in postmenopausal women supplemented with calcium and trace minerals. J Nutr 1994;124:1060-4. 